\title{
Double-contrast Barium Enema
}

National Cancer Institute

\section{Source}

National Cancer Institute. Double-contrast Barium Enema. NCI Thesaurus. Code $C 93005$.

The use of use of positive and negative contrast agents to increase the sensitivity of a radiog raphic study of the colon and/or rectum. 